\title{
A targeted DNA vaccine encoding Fas ligand defines its dual role in the regulation of experimental autoimmune encephalomyelitis
}

\author{
Gizi Wildbaum, ${ }^{1}$ Juergen Westermann, ${ }^{2}$ Gila Maor, ${ }^{3}$ and Nathan Karin ${ }^{2,3}$ \\ ${ }^{1}$ Department of Immunology, and \\ ${ }^{2}$ Rappaport Family Institute for Research in the Medical Sciences and Department of Morphological Sciences, \\ Bruce Rappaport Faculty of Medicine, Technion, Haifa, Israel \\ ${ }^{3}$ Center of Anatomy, Medical School of Hannover, Hannover, Germany \\ Address correspondence to: Nathan Karin, Rappaport Family Institute for Research in the Medical Sciences and \\ Department of Morphological Sciences, Bruce Rappaport Faculty of Medicine, Technion, PO Box 9649, \\ Haifa 31096, Israel. Phone: 972-4-829-5232; Fax: 972-4-851-7797; E-mail: nkarin@tx.technion.ac.il. \\ Received for publication October 22, 1999, and accepted in revised form July 25, 2000.
} This study used naked DNA vaccination to induce breakdown of tolerance to self and thus elicit
immunological memory to native, membrane-bound Fas ligand (FasL). Upon induction of experi-
mental autoimmune encephalomyelitis (EAE), this memory was turned on to provide protective
immunity. FasL-specific autoantibodies isolated from protected animals differentially downregulat-
ed the in vitro production of TNF- $\alpha$, but not IFN- $\gamma$, by cultured T cells. These autoantibodies were
highly protective when they were administered to rats at the onset of EAE. In contrast, administration
of these FasL-specific Ab's to EAE rats after the peak of the acute phase of disease prevented sponta-
neous recovery from disease. This extended illness is partially explained by inhibition of mononuclear
cell apoptosis at the target organ, which resulted in increased accumulation of T cells and
macrophages at the site of inflammation. Hence, FasL exerts two distinct, stage-specific regulatory
functions in the control of this T-cell mediated autoimmune disease of the central nervous system.

J. Clin. Invest. 106:671-679 (2000).

\section{Introduction}

Fas ligand (CD95L; FasL), a type II transmembrane protein that belongs to the TNF family, plays an essential role in both cytotoxicity mediated by cytotoxic $\mathrm{T}$ lymphocytes (CTLs) and homeostasis of the immune system $(1,2)$. In both capacities, FasL functions by engaging Fas (CD95), a member of the TNF-receptor family, and triggering apoptosis in the target cell (3). Fas is constitutively expressed in many different tissues (4), whereas FasL is expressed primarily by activated T cells and natural killer $(\mathrm{NK})$ cells $(3,5)$.

Experimental autoimmune encephalomyelitis (EAE) is a T cell-mediated autoimmune disease of the central nervous system (CNS) that serves as a model for human multiple sclerosis (MS). In both diseases, circulating leukocytes penetrate the blood-brain barrier and damage myelin, resulting in impaired nerve conduction and paralysis (6-9). The role of various proinflammatory and regulatory cytokines in the manifestation and regulation of disease has been widely explored. Proinflammatory cytokines, such as TNF- $\alpha$, play a pivotal role in the initiation and progression of the autoimmune inflammatory process, whereas IL-4, IL-10, and TGF- $\beta$ are implicated in disease regulation. Thus, abrogation of TNF- $\alpha$ proinflammatory activities by either neutralizing Ab's (10), soluble receptor therapy (11), or alteration of the Th1/Th2 balance toward high IL-4-, low TNF- $\alpha$-producing $\mathrm{T}$ cells could prevent, or even reverse, an ongoing disease $(12,13)$. Recent studies have demonstrated that the interaction of FasL with its counterreceptor plays an important role in the development and regulation of autoimmunity (14-22). On one hand, the Fas-FasL interaction has been shown to be essential for maintaining cellular homeostasis in the immune system $(2,23,24)$. Thus, mice with a mutation in the Fas gene (lpr) suffer from a systemic lupus erythematosus-like (SLE-like) autoimmune disease, which probably results from a failure in negative selection of self-reactive T cells (22). On the other hand, Fas- or FasL-deficient mice (lpr or gld mice) were found to be highly resistant or to manifest an ameliorated form of EAE (20, 21). Moreover, FasL-transgenic NOD mice showed heightened sensitivity to diabetogenic T cells, due to self-destruction of beta cells upon T cell-mediated induction of Fas, whereas Fas-negative NOD $(l p r / l p r)$ animals were resistant to diabetogenic $\mathrm{T}$ cells and to spontaneous diabetes $(16,17)$. Thus, accumulating data obtained in transgenic and genetically deficient mice suggest that Fas-FasL interaction may display more than one role in the regulation of self-specific immunity in general and $\mathrm{T}$ cell-mediated autoimmunity in particular $(14,15)$.

Recently, we used the novel technology of naked DNA vaccination to generate self-specific immunity against various $\mathrm{C}-\mathrm{C}$ chemokines of interest and thus prevent the development of EAE (25). The subsequent 
administration of MIP- $1 \alpha$ or MCP- 1 DNA generated self-specific immunity against the product of each construct. Each self-specific Ab titer accelerated in accordance with the progression of disease in control EAE rats and regressed after recovery. These Ab's were neutralizing in vitro and capable of transferring a high state of EAE resistance. The current study uses a similar strategy to generate FasL-specific immunity and evaluate the role of FasL in the regulation of EAE.

\section{Methods}

Rats. Female Lewis rats, approximately 6 weeks old, were purchased from Harlan Laboratories Ltd. Israel (Jerusalem, Israel) and maintained under specific pathogen-free conditions in our animal facility.

Peptide antigens. Myelin basic protein (MBP) p68-86, Y G S L P Q K S Q R S Q D E N P V, was synthesized and purified as described elsewhere (13). Only peptides that were greater than $95 \%$ pure were used in our study.

Immunizations and active disease induction. Active EAE was induced as described elsewhere (13). Rats were then monitored daily for clinical signs by an observer blind to the treatment protocol. EAE was scored as follows: 0 , clinically normal; 1 , flaccid tail; 2 , hindlimb paralysis; 3, total hindlimb paralysis, accompanied by an apparent front-limb paralysis; 4, total hindlimb and front-limb paralysis.

T-cell lines and induction of transferred EAE. MBP p68-86 $\mathrm{T}$-cell line was selected as we described elsewhere (25). Transferred EAE was induced by immunizing Lewis rats intraperitoneally with $10^{7}$ in vitro-activated (day 3) T cells from the encephalitogenic line L68-86. EAE was scored as described above.

Production of FasL construct and DNA vaccination. First, RT-PCR analysis was used on brain samples according to the protocol we described elsewhere (25). FasL specific oligonucleotide primers were designed based on its published sequence (NCBI accession number U03470) as follows: rat Fas ligand sense 5'-CATGCAGCAGCCCGTGAATTACCC-3', rat Fas ligand antisense $5^{\prime}$-TTAAAGCTTATATAAGCCAAAAAAGGT-3'. After cloning and sequencing of PCR products (25), cDNA encoding rat Fas ligand was transferred into a pcDNA3 vector (Invitrogen, San Diego, California, USA) and used as a construct for DNA vaccination as described elsewhere (25).

Production and purification of recombinant FasL. FasL PCR product was recloned into a $\mathrm{PQE}$ expression vector, expressed in Escherichia coli (QIAGEN, Chatsworth, California, USA) and then purified by an NI-NTA-supper flow affinity purification of $6 \times$ His proteins (QIAGEN). After purification, the purity of recombinant FasL was verified by gel electrophoresis. The recombinant protein sequence was verified $\left(\mathrm{NH}_{2}\right.$-terminus) by our sequencing services unit.

Evaluation of anti-FAS ligand Ab titer in sera and spinal cord fluid of DNA-vaccinated rats. A direct ELISA assay was used to determine the anti-Fas ligand $A b$ titer in sera and spinal cord fluid (SCF) from DNA-vaccinated rats. SCF was obtained as described in detail elsewhere (26).
The recombinant Fas ligand, which we produced, was coated onto 96-well ELISA plates (Nunc, Roskilde, Denmark), at concentrations of $50 \mathrm{ng} /$ well. Rat antisera, in serial dilutions from $2^{8}$ to $2^{30}$, were added to ELISA plates. Goat anti-rat IgG alkaline phosphataseconjugated Ab's (Sigma Chemical Co., St. Louis, Missouri, USA) were used as a labeled $\mathrm{Ab}$, and $p$-nitrophenyl phosphate (p-NPP) (Sigma Chemical Co.) was used as a soluble alkaline phosphatase substrate. Results are shown as $\log _{2} \mathrm{Ab}$ titer plus or minus SE.

CNBr purification of FasL-specific Ab's. Recombinant rat FasL (5 mg) was bound to a CNBr-activated Sepharose column according to the manufacturer's instructions (\#17-0820-01; Amersham Pharmacia Biotech, Piscataway, New Jersey, USA). FasL-specific Ab's from sera (IgG fraction) of DNA-vaccinated rats were loaded on the column and then eluted by an acidic elution buffer (glycine, $\mathrm{pH}$ 2.5). Isotype determination of the purified $\mathrm{Ab}$ (ELISA) revealed that purified Ab's are mostly of the IgG2a isotype (data not shown).

Western blot analysis. Plasma membranes were obtained from freshly isolated or 24-hour-activated spleen cells as described elsewhere (27). Proteins were then separated by an SDS-PAGE under reducing conditions and subjected to Western blot analysis according to the protocol described in detail elsewhere (28) with the minor modification of using an $12 \%$ (rather than $8 \%$ ) running gel. CNBr-purified anti-FasL Ab generated by naked DNA vaccination, IgG from normal rat serum (final concentration of $10 \mu \mathrm{g} / \mathrm{mL}$ each), and the commercially available FasL-specific mAb (rat anti-mouse/rat clone H11, \#804-010-c100, at a final concentration of 1:10,000; Alexis Biochemicals, San Diego California, USA) were used as primary Ab's. The rat IgG2a isotype dominates the Ab titer in DNA-vaccinated rats (data not shown), as does clone H11. Goat anti-rat alkaline phosphate-conjugated Ab (Sigma Chemical Co.) was used in a second step. BCIP $(0.15 \mathrm{mg} / \mathrm{mL}$; Sigma Chemical Co.) and nitro blue tetrazolium $(0.3 \mathrm{mg} / \mathrm{mL}$; Sigma Chemical Co.) were then used as a substrate.

FACS analysis. Cells were washed once in FACS buffer (PBS, $0.25 \%$ BSA, $0.05 \%$ sodium azide), and then incubated for 0.5 hour in FACS buffer enriched with $1 \%$ normal rat serum. Then cells were resuspended $\left(4^{\circ} \mathrm{C}\right.$, 0.5 hours) in 96 -well $U$ plates $\left(10^{6} /\right.$ well) with $10 \mu \mathrm{L}$ FACS buffer supplemented with $0.5 \mu \mathrm{g} / \mathrm{mL}$ of either anti-FasL self-specific Ab's generated in DNA-vaccinated rats (CNBr-purified IgG, as described above) or normal rat IgG. Cells were then washed three times and incubated $\left(4^{\circ} \mathrm{C}, 0.5\right.$ hours) with $50 \mu \mathrm{L}$ of FACS buffer supplemented with goat anti-rat IgG-FITC (\#F6258, 1:10,000 dilution; Sigma Chemical Co.). Cells were then washed twice and analyzed in the presence of propidium iodide (PI) using a FACScalibur (Becton Dickinson, Mountain View, California, USA). Data were collected for 10,000 events and analyzed using a Cell Quest program (Becton Dickinson).

Cytokine determination in cultured primary spleen cells. Spleen cells from EAE donors were stimulated in vitro 
(107 cells $/ \mathrm{mL}$ ) in 24-well plates (Nunc) with $100 \mu \mathrm{M}$ p68-86. After 72 hours of stimulation, supernatants were assayed for the protein level of various cytokines using the commercially available ELISA kits we have specified elsewhere $(13,29)$.

Measurement of TNF production by activated macrophages. Levels of TNF- $\alpha$ in macrophage supernatants were determined as described previously (30). Briefly, thioglycollate-elicited peritoneal macrophages were plated in a $96-\mathrm{U}$ plates at a concentration of $5 \times 10^{5}$ cells/well and incubated in the presence of $1 \mu \mathrm{g} / \mathrm{mL}$ LPS (Salmonella enteritidis, L6011; Sigma Chemical Co.) at $37^{\circ} \mathrm{C}, 5 \%$ $\mathrm{CO}_{2}$, for 24 hours. TNF- $\alpha$ levels were determined using a commercial semi-ELISA kit (Genzyme, Cambridge, Massachusetts, USA).

Histopathology and immunohistochemistry. Histological examination of hematoxylin and eosin-stained sections of formalin-fixed, paraffin-embedded sections of the lower thoracic and lumbar regions of the spinal cord was performed. Each section was evaluated without knowledge of the treatment status of the animal. The following scale was used: 0 , no mononuclear cell infiltration; 1 , one to five perivascular lesions per section with minimal parenchymal infiltration; 2 , five to ten perivascular lesions per section with parenchymal infiltration; and 3, more than ten perivascular lesions per section with extensive parenchymal infiltration. The mean histological score plus or minus SE was calculated for each treatment group. Immunohistochemical evaluation was done on frozen sections of the lower thoracic and lumbar regions of the spinal cord, as described by J. Westermann (31). Ab's used for cell staining were as follows: monocytes, ED1; T cells, R73; B cells, His14; NK cells, 3.2.3; and neutrophils, RP1 (31).

In situ apoptosis assay and histomorphometric determination of terminal deoxynucleotidyl transferase-mediated dUTP nick-end labeling-positive cells. A commercially available terminal deoxynucleotidyl transferase-mediated (TdT-mediated) dUTP nick-end labeling (TUNEL) kit (Boehringer Mannheim, Ottweiler, Germany) has been used to assess the extent and localization of apoptosis in situ as descried in detail elsewhere (32). Histomorphometric determination of TUNEL-positive cells was preformed using Olympus Cue-2 image analysis system with appropriate morphometric software (Olympus, Lake Success, New York, USA). Measurements were made in standardized fields with an area of 500,000 $\mu \mathrm{m}^{2}$. In each experimental group nine sections from three different spinal cords were analyzed (two fields per section). Results are shown as the mean of 18 samples \pm SE. Double-staining immunohistological analysis was applied to characterize TUNEL-positive apoptotic cells as follows: Ab 8-18C5 directed against oligodendroglia glycoprotein (kindly provided by C. Linington, Max-Planck Institute, Munich, Germany) was used to stain oligodendrocytes (33). Various leukocyte subtypes were stained using cell-surface markers as described above.
Statistical analysis. Significance of differences was examined using Student's $t$ test. Mann-Whitney rank sum test was used to evaluate the significance of differences in mean of maximum clinical score. Values of $P$ less than 0.05 were considered to be significant.

\section{Results}

FasL-encoding DNA vaccine blocks the development of EAE. Rats were subjected to four weekly injections of FasLencoding DNA vaccines. Control rats were either injected with the pcDNA3 vector alone or with PBS. Two months after the last immunization, all rats were immunized with p68-86/CFA to induce active EAE. All control (PBS immunized) and pcDNA3-vaccinated rats developed active disease that persisted for 5-6 days (Figure 1; six of six in each group with a maximum clinical score of $2.83 \pm 0.18$ in control and $2.33 \pm 0.23$ in pcDNA3-immunized rats). In contrast, rats injected with the FasL naked DNA vaccine developed a markedly reduced degree of disease (Figure 1; incidence of six of six with a maximum clinical score of $1.16 \pm 0.18$, $P<0.016$, and $P<0.0002$ for the comparison of this treatment with either pcDNA3 alone or PBS). Thus, the autoimmune response subsequent to administration of in vivo FasL naked DNA vaccine leads to a marked reduction in disease manifestation. No difference could be observed between the MBP p68-86-specific T-cell proliferative response developed in draining lymph node cells (DLNC) of rats subjected to FasL DNA vaccination and those administered the pcDNA3 vector alone (day 9 of disease, $S I=3.8 \pm 0.3$ vs $3.6 \pm 0.4$ ). Thus, inhibition of EAE in FasL DNA-vaccinated rats (Figure 1) could not be explained by a reduction in antigen-specific T-cell response.

FasL-encoding DNA vaccine induces breakdown of tolerance to its gene product and generates immunity to native membrane-bound FasL. DNA vaccination can potentially elicit both cellular and humoral responses against

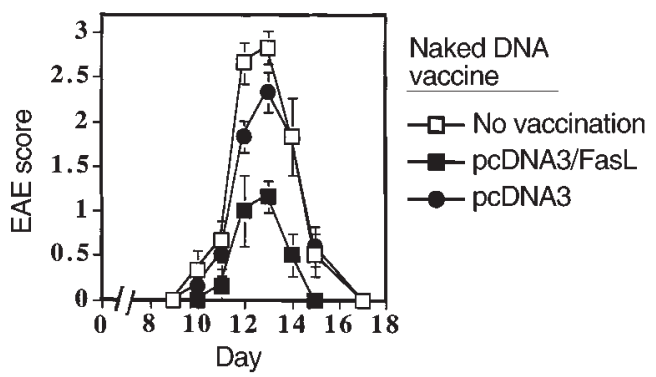

Figure 1

Targeting FasL activity by self-specific naked DNA ameliorates EAE. Rats were immunized weekly with the cloned PCR products of Fas $L$ ligated into a PcDNA3 eukaryotic expression vector, with the pcDNA3 vector alone, or with PBS. Two months after the last immunization, all rats were immunized with p68-86/CFA to induce active EAE and were monitored for clinical signs daily by an observer blind to the treatment protocol. Results are shown as mean clinical score of six rats per group $\pm \mathrm{SE}$. 
a

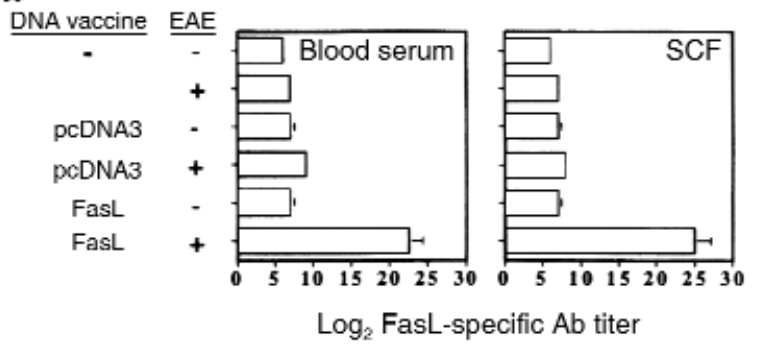

b

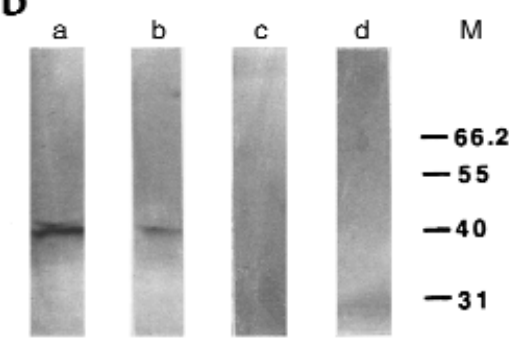

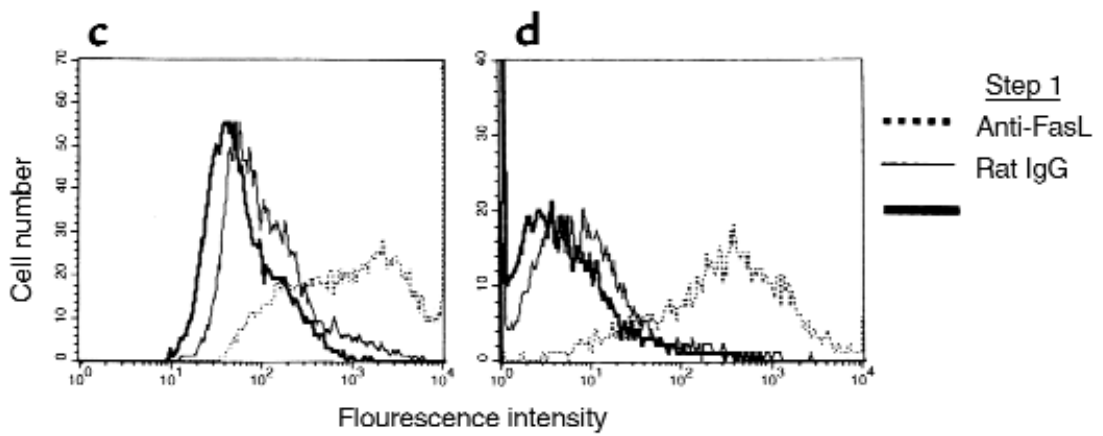

$\underline{\text { Step } 2}$

Goat anti-rat FITC

Goat anti-rat FITC

Goat anti-rat FITC

\section{Figure 2}

FasL-specific Ab's generated in DNA-vaccinated rats bind native FasL. Lewis rats were subjected to four weekly injections of the FasL DNA construct as described in Figure 1. Two months after the last immunization, when FasL-specific Ab titer retained a base line, these rats, as well as rats treated with either the PCDNA3 alone or PBS, were injected with p68-86/CFA to induce active EAE and were sacrificed at different time points. Blood serum and SCF from these rats were analyzed for the presence of Ab's to FasL. (a) $\log _{2}$ Ab titers determined at the onset of disease (day 13). Results are shown as mean of three samples from different rats \pm SE. (b) Plasma membrane proteins were obtained from nonactivated (lane c) or 24-hour-activated (lanes a, b, and d) splenic T cells. SDS-PAGE under reducing conditions was used to separate membrane proteins that were then subjected to Western blot analysis using either a commercially available (see Methods) FasL-specific Ab (lane a), our purified Ab's obtained by naked DNA vaccination (lanes b and c), or control IgG from normal rat serum (lane d). (c and d) Binding of self-specific anti-FasL obtained by naked DNA vaccination to their target receptor (membrane-bound FasL) was determined by flow-cytometry analysis on activated MBP-specific T cells (c) and thioglycollate-elicited peritoneal macrophages (d).

products of a given construct. To determine whether the administration of FasL-DNA vaccines leads to a breakdown of tolerance to self, Lewis rats were subjected to four weekly injections of the FasL-DNA construct as described in Figure 1. Two months after the last immunization, when FasL-specific Ab titer retained a base-line level, these rats, and rats treated with either the pcDNA3 alone or PBS, were injected with p68-86/CFA to induce active EAE. Representative rats from each group were injected with CFA alone. At different time points blood serum and SCF from representative rats were analyzed for the presence of $A b$ 's to FasL. An elicited titer to the above gene product was observed both in blood serum and SCF of FasL DNA-vaccinated rats 8 days after disease induction. This titer reached its maximal level on day 13 (Figure 2a; $P<0.001$ for the compression of $\log _{2} \mathrm{Ab}$ titer for each control group compared with the respective FasL DNA-vaccinated rat). Taken together, our results suggest that FasL naked DNA vaccination leads to a breakdown of immunological tolerance, resulting in the generation of an immunological memory that is turned on upon EAE induction (Figure 2a). CFA immunization alone significantly increased the production of self-specific Ab's to FasL in DNA-vaccinated rats $(P<0.01)$, though to a much lesser extent than did an induction of active disease. The immunological basis of these differences is not yet fully understood and is discussed below.

We then determined whether the above self-specific Ab's bind native FasL. Plasma membrane proteins were obtained from either nonactivated (Figure $2 b$, lane c) or 24-hour activated (Figure 2b, lanes a, b, and d) splenic $T$ cells. Western blot analysis revealed that both the commercially available Ab's and those elicited by DNA vaccination bound a $40-\mathrm{kDa}$ protein that corresponds to native membrane-bound FasL (Figure $2 b$, lanes $a$ and $b$ ). Binding was restricted to activated $\mathrm{T}$ cells (Figure $2 \mathrm{~b}$, lane $\mathrm{b}$ vs. c). Flow cytometry analysis was applied to verify competence of the Ab's above to bind FasL on MBP-activated $\mathrm{T}$ cells from our encephalitogenic T-cell line (Figure 2c) and thioglycollate-elicited peritoneal macrophages that were activated in vitro by LPS (Figure $2 \mathrm{~d}$ ). In accordance with the Western blot analysis, these self-specific Ab's profoundly bound activated cells of both types. Thus, self-specific Ab's generated in DNA-vaccinated rats not only bind recombinant FasL, to which they had been immunized (Figure 2a), but also its natural, membrane-bound form (Figure 2, b-d). We then further evaluated the in vivo and in vitro characteristics of these Ab's. 

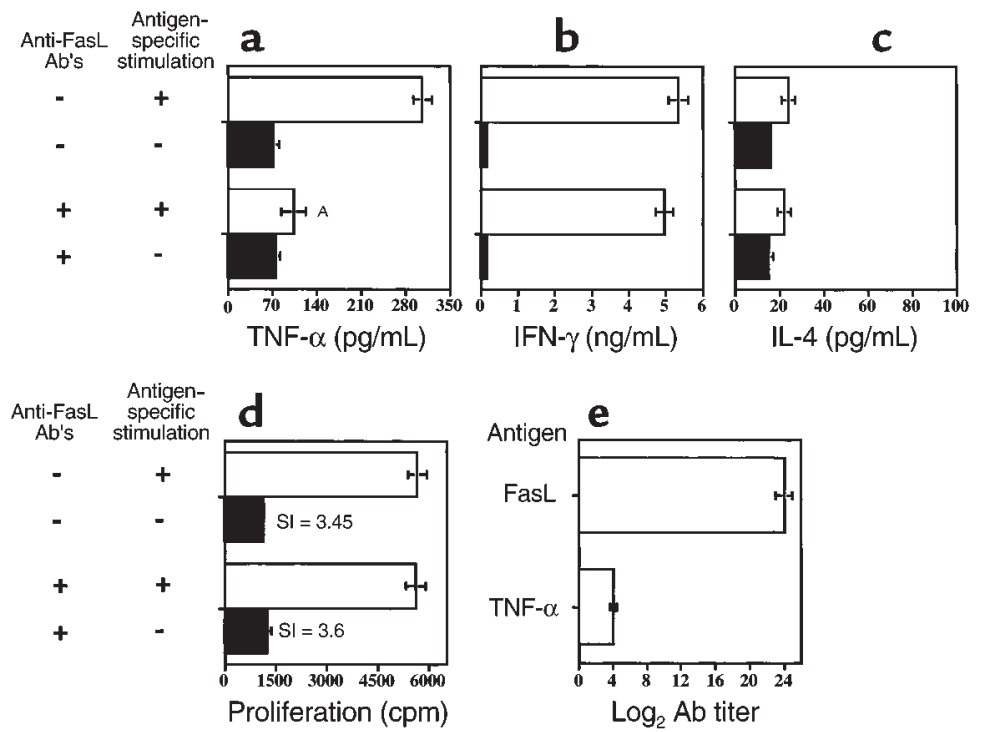

Figure 3

FasL-specific Ab's generated in DNA-vaccinated rats significantly reduce TNF- $\alpha$ production during antigen-specific primary response. Spleen cells from p68-86/CFAprimed (day 9) Lewis rats were cultured with or without the addition of $100 \mu \mathrm{M}$ of MBP p68-86. Cultured cells received $100 \mathrm{ng} / \mathrm{mL}$ of FasL-specific Ab's (IgG from serum of FasL DNA-vaccinated EAE rats [Figure 1, day 13] purified on FasL CNBr-activated Sepharose column) or control IgG from naive rats, as indicated. After 72 hours of stimulation, TNF- $\alpha(\mathbf{a}), \mathrm{IFN}-\gamma(\mathbf{b})$, and IL-4 (c) were determined. The effect of these Ab's on the primary T-cell response to $100 \mu \mathrm{M}$ of MBP p68-86 (d) was determined by an in vitro proliferation assay. Antigen specificity of these Ab's (e) was determined by a direct ELISA assay. The recombinant Fas ligand, which we produced, or commercially available TNF- $\alpha$ was coated onto 96-well ELISA plates at concentrations of $50 \mathrm{ng} /$ well. Rat anti-sera, in serial dilutions from $2^{8}$ to $2^{30}$, were added to ELISA plates. Goat anti-rat IgG alkaline phosphatase-conjugated Ab's were used as a labeled $A b$ and $\mathrm{p}-\mathrm{NPP}$ was used as a soluble alkaline phosphatase substrate. Results are shown as mean of triplicates \pm SE. ${ }^{A} P<0.001$.

FasL-specific Ab's generated in DNA-vaccinated EAE rats significantly reduce TNF- $\alpha$ production during antigen-specific primary response. The addition of FasL self-specific Ab's to MBP-specific (p68-86) cultured spleen cells led to a marked decrease in TNF- $\alpha$ production (Figure $3 \mathrm{a}, P<$ $0.001)$, but not in the production of either IFN- $\gamma$ (Figure $3 \mathrm{~b})$, IL-4 (Figure 3c), or IL-10 (not shown). The addition of these Ab's to the cultured spleen cells had no significant effect on antigen-specific T-cell proliferative responses (Figure 3d, SI $=3.45$ vs. 3.6). These results are remarkable since our FasL self-specific Ab's exhibit very low

\section{Figure 4}

FasL-specific Ab's generated in DNA-vaccinated rats significantly reduce TNF- $\alpha$ transcription and production in LPS-activated macrophages. Thioglycollate-elicited peritoneal macrophages were activated (in vitro) by LPS, with or without the addition of total IgG from naive rat or purified FasL-specific Ab's obtained as described in the legend to Figure 3. Twenty-four hours later, supernatants were collected and levels of TNF- $\alpha$ were determined. Results of triplicates are shown as mean $\pm \mathrm{SE}(\mathbf{a})$. Additionally, at this time mRNA was extracted and subjected to RT-PCR analysis of TNF- $\alpha$ and IL-18 mRNA transcription (b), as described elsewhere $(13,29)$. cross-reactivity to TNF- $\alpha$ (Figure $3 e ; \log _{2} \mathrm{Ab}$ titer of 24 vs. $4, P<0.0001)$. During an antigen-specific immune response, TNF- $\alpha$ is produced not only by activated $T$ cells but also by macrophages. To elucidate the direct effect of the FasL self-specific Ab's on TNF$\alpha$ production by activated macrophages, thioglycollate-elicited peritoneal macrophages were activated (in vitro) by LPS, with or without the addition of either control IgG from naive Lewis rats or with purified FasL-specific Ab's (Figure 4). Only the addition of FasL self-specific Ab's led to a significant reduction in TNF- $\alpha$ production by these cells (Figure 4, $990 \pm 48$ vs. $720 \pm 40$ $\mathrm{pg} / \mathrm{mL}$, with backgrounds of $620 \pm 34$ and $630 \pm 42 \mathrm{pg} / \mathrm{mL}$, respectively; $P<0.01$ ). These Ab's did not affect the viability of cultured macrophages (as determined by trypan blue uptake). Thus, the reduction in TNF- $\alpha$ production in the presence of FasLspecific Ab's could not be explained by a possible loss of viability of macrophages. To determine whether the reduction above could be attributed to a direct effect on TNF- $\alpha$-specific mRNA transcription, the experiment described above (Figure 4a) was repeated once again, and levels of mRNA encoding either TNF- $\alpha$ or IL-18 were determined as we describe in detail elsewhere (13, 29). It appears that cultured macrophages exhibited a marked reduction in TNF- $\alpha$, but not in IL-18, mRNA transcription (Figure $4 \mathrm{~b})$. This may explain the significant reduction in TNF- $\alpha$ production in these cells (Figure 4a) and possibly in primary cultured spleen cells (Figure 3a). Similar results were also obtained using a commercially available Ab (clone H11; Alexis Biochemicals). It is not yet clear whether the binding of soluble FasL to its receptor enhances TNF- $\alpha$ mRNA transcription or if docking membrane-bound FasL by the antiFasL Ab initiates a signal transduction cascade resulting in reduced TNF- $\alpha$ mRNA transcription.

Administration of FasL-specific Ab's during the early stage of disease confers EAE resistance. The purified FasL-specific $A b$ 's were then evaluated for their competence to trans-

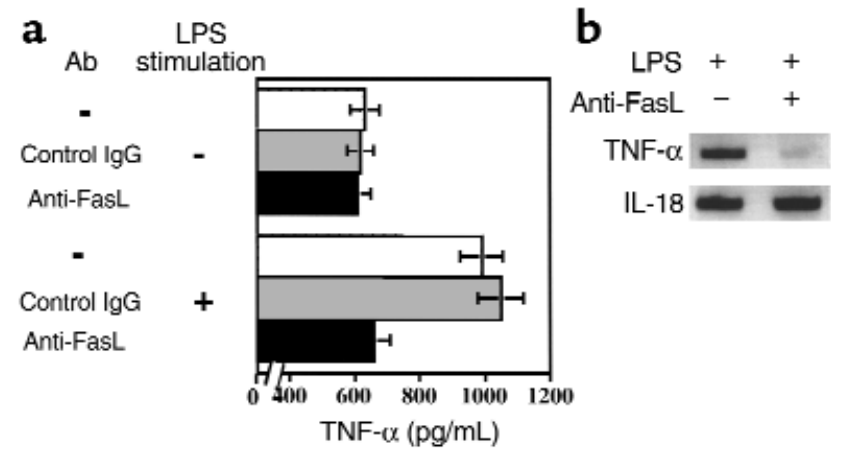




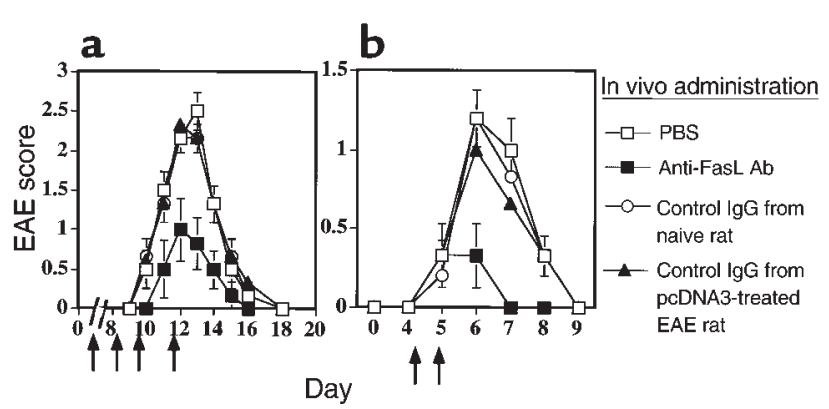

Figure 5

Administration of FasL-specific Ab's during the early stage of disease confers resistance to EAE. (a) Lewis rats were immunized with p6886/CFA to develop active EAE (day 0 ). At various time points (days 7 , 9,10 , and 12) these rats were administered $100 \mu \mathrm{g} /$ rat of purified FasL-specific Ab's, obtained as described in the legend to Figure 3. Control rats were administered either IgG from rats that were vaccinated with pcDNA3 and then subjected to active induction of disease (Figure 1, day 13), or with IgG from naive Lewis rats. All rats were then monitored for clinical signs daily by an observer blind to the treatment protocol. Results are shown as mean clinical score of six rats per group \pm SE. (b) Lewis rats were administered L68-86 to induce transferred EAE (day 0 ). On days 4 and 5 these rats were administered 100 $\mu \mathrm{g} / \mathrm{rat}$ of each of the Ab's described in a. All rats were monitored for clinical signs daily by an observer blind to the treatment protocol. Results are shown as mean clinical score of six rats per group $\pm \mathrm{SE}$.

fer protection against active (Figure 5a) and transferred (Figure 5b) EAE. The subsequent administration of these Ab's starting 1-3 days before the onset of disease led to a marked reduction in the clinical (Figure 5, a and b) and histological (Figure 6) score of disease. That is, all control EAE rats immunized with p68-86/CFA and then treated with either PBS or with IgG (protein G purified) from either naive or $\mathrm{pcDN} 3$-treated $\mathrm{EAE}$ rats developed severe EAE (Figure 5a; mean maximal score of $2.5 \pm 0.24$, $2.33 \pm 0.23$, and $2.33 \pm 0.23$, respectively). In contrast, those treated with purified FasL-specific Ab's exhibited a mild form of disease (mean maximal score of $0.833 \pm$ $0.3, P<0.001$, compared with each control group). Disease inhibition was accompanied by a significant, but not total, reduction in parenchymal mononuclear cell infiltration (Figure $6 \mathrm{~d}$ vs. Figure $6, \mathrm{~b}$ and c, mean histological scores of $1 \pm 0$ vs. $2.3 \pm 0.2$ in each control group). To assess the possibility that the administration of FasLspecific Ab's affected apoptosis in the CNS, we analyzed EAE lesions for DNA fragmentation within dying cells by using the TUNEL assay. CNS sections from a naive brain with no mononuclear infiltration (Figure 6a) did not show any TUNEL-positive cells. In contrast, sections from EAE brains of rats that were treated with either PBS or control IgG and that exhibited a substantial parenchymal mononuclear cell infiltration (Figure 6, b and c), displayed many TUNEL-positive cells in perivascular inflammatory cuffs and adjacent white matter (630 \pm 45 and $570 \pm 60$ positive cells $/ \mathrm{mm}^{2}$; Figure 6 , f and g, demonstrate representative sections). However, a marked reduction in the number and intensity of apoptotic cells could be found in CNS sections from rats that were treated with FasL-specific Ab's (less than 40 positive cells $/ \mathrm{mm}^{2}$. Figure $6 \mathrm{~h}$ demonstrates a representative section, $P<0.001$ compared to Figure $6 \mathrm{f}$ and Figure $6 \mathrm{~g}$ ). Interestingly, at this stage of disease ( 2 days after its onset), apoptotic mononuclear infiltrating cells could hardly be detected in any of these sections (Figure 6, e-h), suggesting that FasL-specific immunotherapy prevents the apoptosis of target CNS cells.

Administration of FasL-specific Ab's in the late stage of disease delays recovery from the acute phase. Lewis rats were immunized with p68-86/CFA to develop active EAE (day 0$)$. Ten days later, all rats (six of six per group) manifested apparent signs of paralysis (Figure 7, score $1 \pm 0$ in all groups). At various time points (days 12, 13, and 14) these rats were administered purified FasL-specific Ab's, obtained as described in the legend to Figure 3. Control rats were administered either IgG from rats

\section{Figure 6}

CNS histology and in situ apoptosis after early administration of FasL-specific Ab's. Twelve days after active induction of EAE, representative rats from each of the groups described in the legend to Figure 5a were sacrificed. Sections of the lower thoracic and lumbar regions of the spinal cord of three different rats per group were subjected to histological (a-d) and in situ TUNEL staining $(\mathbf{e}-\mathbf{h})$. Each section was evaluated without knowledge of the treatment status of the animal. Representative staining from each group is shown $(\times 40)$ as follows: $\mathbf{a}$ and $\mathbf{e}$, naive rat; $\mathbf{b}$ and $\mathbf{f}$, control PBS-treated EAE rat; $\mathbf{c}$ and $\mathbf{g}$, control IgG-treated EAE rat; $\mathbf{d}$ and $\mathbf{h}$, antiFasL-treated EAE rat. Histomorphometry analyses were done on 18 different fields from each group and are given in the text. Arrows indicate apoptotic cells.



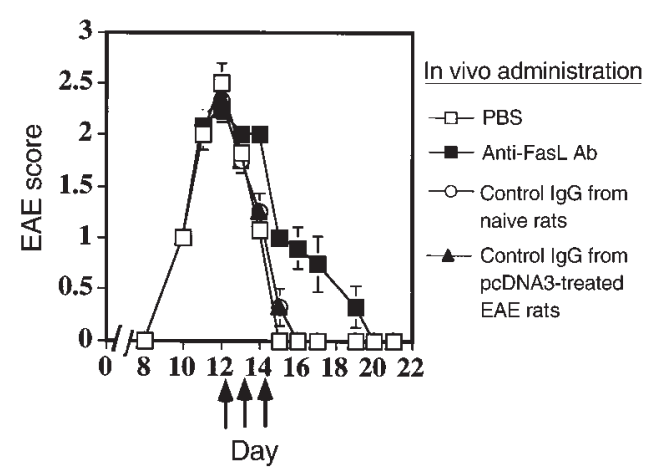

\section{Figure 7}

Administration of FasL-specific Ab's during the late stage of disease delays recovery from its acute phase. Lewis rats were immunized with p68-86/CFA to develop active EAE (day 0 ). At various time points (days 12, 13, and 14) these rats were administered $100 \mu \mathrm{g}$ per rat of either purified FasL-specific Ab's, obtained as described in legend to Figure 3. Control rats were administered either IgG from rats that were vaccinated with pcDNA3 and then subjected to active induction of disease (Figure 1, day 13) or with IgG from naive Lewis rats. All rats were then monitored for clinical signs daily by an observer blind to the treatment protocol. Results are shown as mean clinical score of six rats per group \pm SE.

that were vaccinated with pcDNA3 and then subjected to active induction of disease (Figure 1, day 13) or with IgG from naive Lewis rats (Figure 7). Whereas control rats recovered from the acute phase of disease by day 15, those treated with FasL-specific Ab's continued to manifest clinical paralyses for another 4-5 days (Figure 7). On day 18 , when control rats had all fully recovered, those treated with FasL-specific Ab's still manifested clinical paralysis. Representative rats from each of the groups were sacrificed. Sections of the lower thoracic and lumbar regions of the spinal cord of three rats per group were subjected to histological (Figure $8, a-c$ ) and in situ TUNEL staining (Figure 8, d-f). Control postEAE rats that were treated with PBS or with control IgG exhibited only few infiltrating mononuclear cells per section (Figure 8, a and b), whereas those treated with FasL-specific Ab's displayed an extensive parenchymal mononuclear cell infiltration (Figure 8c). TUNEL staining clearly revealed that in post-EAE rats (Figure 8, $\mathrm{d}$ and e) mononuclear, but not CNS, resident cells undergo extensive apoptosis (Figure 8, $\mathrm{d}$ and e, compared with early stages of disease, Figure 6 , f and g). This could explain, in part, the disappearance of mononuclear cells from the site of inflammation at this stage of disease (Figure 8, a and b, compared with Figure $6, \mathrm{~b}$ and $\mathrm{c})$. In contrast, sections from EAE rats that were subjected to late administration of FasL-specific Ab's show very few TUNEL-positive mononuclear cells (less than $40 / \mathrm{mm}^{2}$ compared with $520 \pm 30$ and $540 \pm 25$ positive cells $/ \mathrm{mm}^{2}$ in nontreated or normal IgG-treated control rats, $P<0.001)$. This may then explain, in part, their apparent accumulation at the site of inflammation (Figure 8c), which correlates with the delay in recovery (Figure 7).
Finally, we have used immunohistochemical analysis of spinal cord samples to distinguish different leukocyte subsets at various stages of active EAE in positive control and anti-FasL-treated rats. In these experiments, double staining was also applied to characterize the TUNEL-positive cells. At the onset of disease (day 9), about $45 \%$ of infiltrating cells were ED1-positive macrophages compared with $20 \%$ R73-positive T cells, 15\% His14-positive B cells, 5\% 3.2.3-positive NK cells, and 15\% RP1-positive neutrophils. At the peak of disease (day 12) and just after recovery (day 15-16), the vast majority of detected leukocytes were ED1-positive macrophages (60\% and $75 \%$, respectively), $20 \%$ and $15 \%$ $\mathrm{T}$ cells, and less than $10 \%$ from each of the other cell types. Interestingly, in rats that were treated at a late stage of disease with anti-FasL Ab's, and therefore displayed a continuing illness (Figure 6), the ratio of ED1positive macrophages and $T$ cells at the site of inflammation was in favor of the $\mathrm{T}$ cells (day 16, 30\% ED1-positive macrophages vs. $45 \%$ positive T cells). Surprisingly, just after recovery (day 15), the vast majority of TUNEL-positive mononuclear cells were ED1-positive macrophages. It has also been shown by others that before recovery, $\mathrm{T}$ cells preferentially undergo apopto-

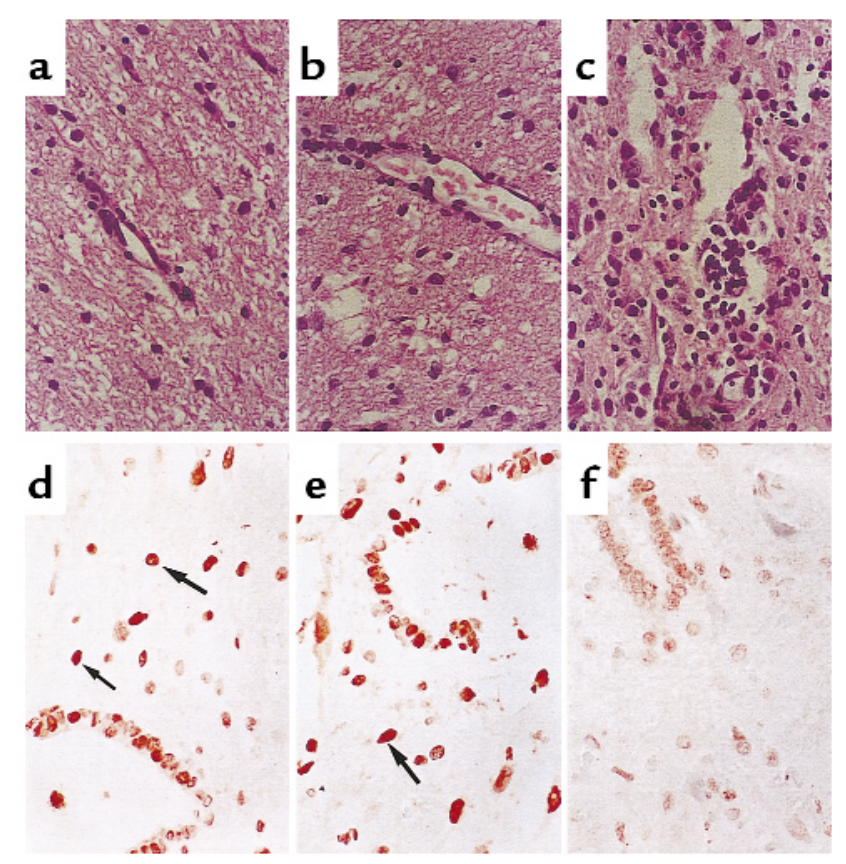

Figure 8

CNS histology and in situ apoptosis after late administration of FasLspecific Ab's. Eighteen days after active induction of EAE, representative rats from each of the groups that are described in the legend to Figure 7 were sacrificed. Sections of the lower thoracic and lumbar regions of the spinal cord of three different rats per group were subjected to histological staining $(\mathbf{a}-\mathbf{c})$ and in situ TUNEL staining (d-f). Each section was evaluated without knowledge of the treatment status of the animal. Representative staining from each group is shown $(\times 40)$ as follows: $\mathbf{a}$ and $\mathbf{d}$, control PBS-treated EAE rat; $\mathbf{b}$ and $\mathbf{e}$, control IgG-treated EAE rat; $\mathbf{c}$ and $\mathbf{f}$, anti-FasL-treated EAE rat. Histomorphometric analyses were done on 18 different fields from each group and is given in the text. Arrows indicate apoptotic cells. 
sis at the site of inflammation $(33,34)$. We therefore suggest that saving $T$ cells and macrophages from apoptosis, at late stages of disease, may provide a milieu for a continuing illness. Interestingly, under these conditions the $\mathrm{T}$-cell/macrophage ratio is in favor of the $\mathrm{T}$ cells. This not only demonstrates the role of FasL in the regulation of the late stage of disease, but also emphasizes the pivotal role of $\mathrm{T}$ cells as the driving force of the inflammatory condition. At the peak of the disease in control EAE rats (day 12), the vast majority of ED1-positive apoptotic cells in spinal cord sections resembled in shape and size CNS resident cells (i.e., microglia), rather than invading monocytes/macrophages (Figure 6, f and g). Using the 8-18C5 mAb directed against oligodendroglia glycoprotein, we could not find compelling evidence to demonstrate direct apoptosis of oligodendrocytes. This further supports recent findings suggesting that in EAE, and probably in MS, loss of oligodendrocytes is due to necrosis (probably induced by glutamate excitotoxicity) rather than apoptosis (35).

\section{Discussion}

This study uses the novel technology of naked DNA vaccination to generate protective immunity against a self gene product (FasL) and thus to interfere with the development of a $\mathrm{T}$ cell-mediated autoimmune disease. Thereafter, it uses self-specific Ab's generated in DNA-vaccinated rats to explore the dual role of FasL in $\mathrm{T}$ cell-mediated autoimmunity.

We have demonstrated recently that naked DNA vaccines that include constructs encoding self-proinflammatory chemokines $(25,36)$ or TNF- $\alpha$ (29), together with an immunostimulatory sequence (ISS) $(37,38)$, may be used to elicit a breakdown of tolerance to self. The current study extends these findings to FasL, a transmembrane protein that also appears as a soluble ligand. Interestingly, the induction of an autoimmune condition elicited the generation of self-specific immunity to this gene product much stronger than CFA immunization alone. The underlying mechanism is not fully understood. A partial explanation for this interesting phenomenon has been suggested previously by C.C. Goodnow and his group (39). This group demonstrated that peripheral clonal exclusion of self-reactive $B$ cells occurs at germinal centers of lymph nodes that drain tissues lacking immune surveillance (i.e., immune-privileged areas), where competition for follicular niches do not exclude self-reactive cells from the recirculating B-cell repertoire (39). Thus, production of proinflammatory cytokines, chemokines, or FasL at the CNS would not lead to clonal exclusion of self-reactive $\mathrm{B}$ cells, as would their production at a site with no immune prevalence. We think that DNA vaccination that enhances an effective breakdown of tolerance to self gene products allows a further amplification of this process. At this point, we cannot exclude the possibility that immunocomplexes, possibly generated during an autoimmune condition, contribute to the elicited $\mathrm{Ab}$ titer determined in DNA-vaccinated EAE rats.
Since EAE resistance in DNA vaccinated rats was associated with production of self-specific Ab's, we have determined their possible contribution to the tolerant state by adoptive transfer experiments. Interestingly, whereas our FasL self-specific Ab's could block the development of EAE when administered before the peak of disease (Figure 5), they delayed recovery when inoculated at a later time (Figure 7). This clearly implies a dual role for FasL in the regulation of EAE and possibly other $T$ cell-mediated autoimmune diseases. Why should an early administration of FasL-specific Ab's prevent EAE?

It has been well documented that Fas-FasL interactions can lead to apoptosis of cells at a target organ. The current study also demonstrates a role of FasL in the regulation of TNF- $\alpha$, a highly important mediator of the inflammatory process in $\operatorname{EAE}(10,11)$ and probably other T cell-mediated autoimmune diseases. Our study demonstrates, we believe for the first time, that anti-FasL Ab's downregulate mRNA transcription on activated macrophages (Figure 4b). Along with this observation, early administration of FasL Ab's led to a significant decrease of TNF- $\alpha$ mRNA transcription at the site of inflammation (day 12, RT-PCR on spinal cord samples; data not shown). Hence, it is not clear what contributed more to disease inhibition, the direct effect of anti-FasL on TNF- $\alpha$ mRNA transcription in activated macrophages (Figure $4 \mathrm{~b}$ ) or the quantitative reduction in the accumulation of macrophages and $\mathrm{T}$ cells at this site (Figure 6). It is conceivable that in addition to the direct blockade of the Fas-FasL interactions, anti- FasL Ab's inhibit the production of TNF- $\alpha$ and thus inhibit EAE. A major difference between these effects is that the Fas-FasL interaction may also include non-MHC class II-expressing resident cells of the CNS and may thus include a bystander response, which may contribute to the destructive effect of this interaction (19). This interaction has also been associated with the apoptosis of non-MHC class II-expressing resident cells of the CNS in MS, as well $(40,41)$.

In contrast to their protective competence, at early stages of disease administration of FasL Ab's after the peak of disease delayed recovery. It has been documented that before recovery $\mathrm{T}$ cells undergo apoptosis at the site of inflammation $(33,34)$. However, the fate of invading macrophages after recovery is still elusive. It has been suggested that at the end of an inflammatory process macrophages do not undergo apoptosis but rather leave the site of inflammation (42). Our data clearly show that after recovery from EAE-invading ED1-positive macrophages do undergo apoptosis and that anti-FasL Ab's administered at that time preserve the continuing persistence of $\mathrm{T}$ cells and macrophages at the site of inflammation, and thus prevent recovery. It is not clear, however, why $\mathrm{T}$ cells and macrophages undergo apoptosis at late stages, but to a much less extent at early stages of disease. It is also not fully understood why FasL Ab's display a different effect on leukocyte apoptosis when administered at different 
time points. A recent work of Zhang et al. (43) showing that different $\mathrm{CD} 4^{+} \mathrm{T}$-cell subtypes display different sensitivity to FasL-mediated apoptosis may provide a partial explanation for the observation above and its clinical implications.

\section{Acknowledgments}

This study was supported by Israel Science Foundation, Israel Cancer Research Fund (ICRF), Israel Chief Ministry of Health, San Francisco Diabetes Research Foundation, the Technion VPR Albert Goodstein research fund, and by the Rappaport Family Institute for Research fund. We would like to thank H. Gershon for creative discussion and for critical reading of the manuscript.

1. Nagata, S., and Goldstein, G. 1995. The FAS death factor. Science 267:1449-1455.

2. Griffith, T.S., Brunner, T., Fletcher, S.M., Green, D.R., and Ferguson, T.A. 1995. Fas ligand-induced apoptosis as a mechanism of immune privilege. Science. 270:1189-1192.

3. Suda, T., et al. 1995. Expression of the Fas ligand in cells of T cell lineage. J. Immunol. 154:3806-3813.

4. Watanabe-Fukunaga, R., et al. 1992. The cDNA structure, expression, and chromosomal assignment of the mouse Fas antigen. J. Immunol. 148:1274-1279.

5. Bossi, G., and Griffiths, G.M. 1999. Degranulation plays an essential part in regulation cell surface expression of Fas ligand in T cells and natural killer cells. Nat. Med. 5:90-96.

6. Alvord, E.C., Jr. 1984. The challenge: how good a model of MS is EAE today? Prog. Clin. Biol. Res. 146:3-5.

7. MacFarlin, D., and MacFarland, H. 1983. Multiple sclerosis. N. Engl. J. Med. 307:1183-1188.

8. Yednock, T.A., et al. 1992. Prevention of experimental autoimmune encephalomyelitis by antibodies against alpha 4 beta 1 integrin. Nature. 356:63-66.

9. Karin, N., Szafer, F., Mitchell, D., Gold, D.P., and Steinman, L. 1993. Selective and nonselective stages in homing of T lymphocytes to the central nervous system during experimental allergic encephalomyelitis. $J$. Immunol. 150:4116-4124.

10. Ruddle, N.H., et al. 1990. An antibody to lymphotoxin and tumor necrosis factor prevents transfer of experimental allergic encephalomyelitis. $J$. Exp. Med. 172:1193-1200.

11. Selmaj, K., Papierz, W., Glabinski, A., and Kohno, T. 1995. Prevention of chronic relapsing experimental autoimmune encephalomyelitis by soluble tumor necrosis factor receptor I. J. Neuroimmunol. 56:135-141.

12. Leonard, J.P., Waldburger, K.E., and Goldman, S.J. 1995. Prevention of experimental autoimmune encephalomyelitis by antibodies against interleukin 12. J. Exp. Med. 181:381-386.

13. Wildbaum, G., Youssef, S., Grabie, N., and Karin, N. 1998. Prevention of experimental autoimmune encephalomyelitis by antibodies to interferon gamma inducing factor. J. Immunol. 161:6368-6374.

14. Suvannavejh, G.C., Dal Canto, M.C., Matis, L.A., and Miller, S.D. 2000 Fas-mediated apoptosis in clinical remissions of relapsing experimental autoimmune encephalomyelitis. J. Clin. Invest. 105:223-231.

15. Sabelko-Downes, K.A., Cross, A.H., and Russell, J.H. 1999. Dual role for Fas ligand in the initiation of and recovery from experimental allergic encephalomyelitis. J. Exp. Med. 189:1195-1205.

16. Chervonsky, A.V., et al. 1997. The role of Fas in autoimmune diabetes. Cell. 89:17-24.

17. Itoh, N., et al. 1997. Requirement of Fas for the development of autoimmune diabetes in nonobese diabetic mice. J. Exp. Med. 186:613-618.

18. Lau, H.T., and Stoeckert, C.J. 1997. FasL: too much of a good thing? Transplanted grafts of pancreatic islet cells engineered to express Fas ligand are destroyed not protected by the immune system. Nat. Med. 3:727-728.

19. Thilenius, A.R.B., Sabelko-Dowens, K.A., and Russell, J.H. 1999. The role of the antigen presenting cell in Fas-mediated direct and bystander killing: potential in vivo function in experimental allergic encephalomyelitis. J. Immunol. 162:643-650.

20. Waldner, H., Sobel, R.A., Howard, E., and Kuchroo, V.K. 1997. Fas and FasL deficient mice are resistant to induction of autoimmune encephalomyelitis. J. Immunol. 159:3100-3103.

21. Sabelko, K.A., Kelly, K.A., Nahm, M.H., Cross, A.H., and Russell, J.H. 1997. Fas and Fas ligand enhance the pathogenesis of experimental allergic encephalomyelitis, but are not essential for immune privilege in the central nervous system. J. Immunol. 159:3096-3099.

22. Watanabe-Fukunaga, R., Brannan, C.I., Copeland, N.G., Jenkins, N.A., and Nagata, S. 1992. Lymphoproliferation disorder in mice explained by defects in Fas antigen that mediates apoptosis. Nature. 356:314-317.

23. Chen, J.J., Sun, Y., and Nabel, G.J. 1998. Regulation of the proinflammatory effects of Fas ligand (CD95L). Science. 282:1714-1717.

24. Nagata, S. 1996. Fas ligand and immune evasion. Nat. Med. 2:1306-1307.

25 . Youssef, S., et al. 1998. Long lasting protective immunity to experimental autoimmune encephalomyelitis following vaccination with naked DNA encoding C-C chemokines. J. Immunol. 161:3870-3879.

26. Gijbels, K., et al. 1993. Gelatinase B is present in the cerebrospinal fluid during experimental autoimmune encephalomyelitis and cleaves myelin basic protein. J. Neurosci. Res. 36:432-440.

27. Silvius, J.R. 1992. Solubilization and functional reconstitution of biomembrane components. Annu. Rev. Biophys. Biomol. Struct. 21:323-348.

28. Hogger, P., Dreier, J., Droste, A., Buck, F., and Sorg, C. 1998. Identification of the integral membrane protein RM3/1 on human monocytes as a glucocorticoid-inducible member of the scavenger receptor cysteinerich family (CD163). J. Immunol. 161:1883-1890.

29. Wildbaum, G., and Karin, N. 1999. Augmentation of natural immunity to a pro-inflammatory cytokine (TNF- $\alpha$ ) by targeted DNA vaccine confers long lasting resistance to experimental autoimmune encephalomyelitis. Gene Ther. 6:1128-1138.

30. Bemelmans, M.H., Gouma, D.J., and Buurman, W.A. 1993. LPS-induced sTNF-receptor release in vivo in a murine model. Investigation of the role of tumor necrosis factor, IL-1, leukemia inhibiting factor, and IFNgamma. J. Immunol. 151:5554-5562.

31. Westermann, J., et al. 1996. Blood leukocyte subsets of the rat: expression of adhesion molecules and localization within high endothelial venules. Scand. J. Immunol. 43:297-303.

32. Bonetti, B., and Raine, C.S. 1997. Multiple sclerosis: oligodendrocytes display cell death-related molecules in situ but do not undergo apoptosis. Ann. Neurol. 42:74-84.

33. Schmied, M., et al. 1993. Apoptosis of T lymphocytes in experimental autoimmune encephalomyelitis: evidence for programmed cell death as a mechanism to control inflammation in the brain. Am. J. Pathol. 143:446-451.

34. Bonetti, B., Pohl, J., Gao, Y.L., and Raine, C.S. 1997. Cell death during autoimmune demyelination: effector but not target cells are eliminated by apoptosis. J. Immunol. 159:5733-5741.

35. Pitt, D., Werner, P., and Raine, C.S. 2000. Glutamate excitotoxicity in a model of multiple sclerosis. Nat. Med. 6:67-70.

36. Youssef, S., Wildbaum, G., and Karin, N. 1999. Prevention of experimental autoimmune encephalomyelitis by MIP-1alpha and MCP-1 naked DNA vaccines. J. Autoimmun. 13:21-29.

37. Raz, E., et al. 1996. Preferential induction of a Th1 immune response and inhibition of specific IgE antibody formation by plasmid DNA immunization. Proc. Natl. Acad. Sci. USA. 93:5141-5145.

38. Sato, Y., et al. 1996. Immunostimulatory DNA sequences necessary for effective intradermal gene immunization. Science. 273:352-357.

39. Cyster, J.G., Hartley, S.B., and Goodnow, C.C. 1994. Competition for follicular niches excludes self-reactive cells from the recirculating B-cell repertoire. Nature. 371:389-395.

40. Dowling, P., et al. 1996. Involvement of the CD95 (APO-1/Fas) receptor/ligand system in multiple sclerosis brain. J. Exp. Med. 184:1513-1518

41. D'Souza, S.D., et al. 1996. Multiple sclerosis: Fas signaling in oligodendrocyte cell death. J. Exp. Med. 184:2361-2370.

42. Bellingan, G.J., Caldwell, H., Howie, S.E., Dransfield, I., and Haslett, C. 1996. In vivo fate of the inflammatory macrophage during the resolution of inflammation: inflammatory macrophages do not die locally, but emigrate to the draining lymph nodes. J. Immunol. 157:2577-2585.

43. Zhang, X., et al. 1997. Unequal death in Thelper cell (Th) 1 and Th2 effectors: Th1, but not Th2, effectors undergo rapid Fas/FasL-mediated apoptosis. J. Exp. Med. 185:1837-1849. 